\title{
Lo mejor del Congreso de la Sociedad Europea de Cardiología 2017
}

\begin{tabular}{|c|c|c|c|}
\hline \multirow[t]{6}{*}{ Palabras clave: } & CONGRESOS & Key words: & CONGRESS \\
\hline & CARDIOLOGÍA & & CARDIOLOGY \\
\hline & CANTOS & & CANTOS \\
\hline & GARFIELD-AF & & GARFIELD-AF \\
\hline & CASTLE-AF & & CASTLE-AF \\
\hline & COMPASS & & COMPASS \\
\hline
\end{tabular}

A pesar de la tragedia que el último atentado terrorista provocó en las ramblas de Barcelona, la comunidad cardiológica catalana tuvo que reponerse rápidamente para ser anfitriona del mayor evento cardiovascular de Europa: el ESC CONGRESS 2017. Del 26 al 30 de agosto, en decenas de mesas redondas, más de 31.000 cardiólogos de todo el mundo se reunieron para actualizar sus conocimientos e intercambiar novedades en ciencia, innovación e investigación en cardiología en más de 500 sesiones y más de 4.500 presentaciones de abstracts ${ }^{(1)}$.

Se presentaron nuevas guías para el manejo del infarto agudo de miocardio, enfermedad valvular, enfermedad arterial periférica y recomendaciones para el uso de terapia antiplaquetaria dual. Se celebraron los 40 años de la intervención coronaria percutánea (PCI), realizada por Andreas Grüntzig por primera vez el 16 de setiembre de 1977(1).

Realizaremos un breve resumen de algunos de los principales trabajos científicos presentados durante este evento que sin duda tendrán un impacto en nuestra práctica clínica diaria.

- Canakinumab Anti-Inflammatory Thrombosis Outcome Study: CANTOS.

- Global Anticoagulant Registry in the Field-Atrial Fibrillation (Registro Global de Anticoagulantes en el Campo de la Fibrilación Auricular): GARFIELD-AF.

- Catheter ablation versus standard conventional treatment in patients with left ventricular dysfunction and atrial fibrillation: CASTLE-AF.

- Cardiovascular OutcoMes for People using Anticoagulation StrategieS (COMPASS) trial: Primary Results 


\section{Canakinumab Anti-Inflammatory Thrombosis Outcomes Study: CANTOS}

Desde hace unos años se tiene conocimiento de que la inflamación juega un rol en el desarrollo de la enfermedad cardiovascular aterotrombótica. Tanto células inflamatorias como sus vías de señalización son necesarias para reparar el daño vascular, permitiendo la iniciación y el crecimiento de la placa aterosclerótica. Existen datos que sugieren que disminuir la inflamación, sin afectar el nivel de lípidos, podría reducir el riesgo cardiovascular, pero no se había podido comprobar esta hipótesis hasta el momento. La interleuquina $1 \beta$ (IL-1 $\beta$ ) es central en la respuesta inflamatoria y dirige el camino de señalización de la interleuquina 6 (IL-6). El canakinumab es un anticuerpo monoclonal humano anti IL-1 $\beta$ que tiene efectos antiinflamatorios y ya ha sido aprobado para su uso en enfermedades reumatológicas. El estudio CANTOS se realizó con el objetivo de demostrar que el empleo de canakinumab podría evidenciar la hipótesis inflamatoria de la enfermedad aterotrombótica.

Se trató de un estudio aleatorizado, doble ciego, controlado, en pacientes estables con antecedentes de infarto agudo de miocardio con una respuesta pro inflamatoria persistente, definida por niveles de proteína C ultrasensible (PCR) mayor a $2 \mathrm{mg} / \mathrm{l}$, para evaluar si canakinumab puede prevenir la recurrencia de eventos vasculares. Se incluyeron 10.061 pacientes de 39 países y se compararon tres dosis de canakinumab (50 mg, $150 \mathrm{mg}$ y $300 \mathrm{mg}$ ) administradas de forma subcutánea cada tres meses versus placebo ${ }^{(2,3)}$.

El objetivo primario de eficacia fue la ocurrencia de infarto agudo de miocardio (IAM) no fatal, accidente cerebrovascular no fatal (ACV) o muerte cardiovascular. Como objetivos secundarios preespecificados: 1) objetivo primario más hospitalización por angina inestable que requiriera revascularización; 2) muerte de cualquier causa, y 3) el compuesto de IAM no fatal, cualquier ACV no fatal y muerte de cualquier cau$\mathrm{sa}^{(2,3)}$.

Los resultados fueron presentados por el principal investigador, el Dr. Paul M Ridker (Brigham and Women's Hospital, Boston, MA) y simultáneamente publicados en New England Journal of Medicine ${ }^{(2)}$ y Lancet $^{(3)}$.

De 17.482 pacientes evaluados se aleatorizaron 10.061. Edad media 61 años, $27,5 \%$ mujeres, $40 \%$ diabéticos y $66,7 \%$ con algún procedimiento de revascularización previo. El 95\% estaba recibiendo tratamiento antitrombótico, 93,4\% hipolipemiante, $91,4 \%$ antiisquémicos y 79,7\% recibía inhibidores del sistema renina-angiotensina-aldosterona. La mediana de la PCR fue de 4,2 mg/l y la mediana del LDL-C fue de $82,4 \mathrm{mg} / \mathrm{dl}^{(2-4)}$.

A los 48 meses la reducción media de la PCR fue $26 \%$ mayor en el grupo que recibía $50 \mathrm{mg}$ de canaki- numab, 37\% mayor en el grupo que recibía $150 \mathrm{mg}$ y $41 \%$ mayor en el grupo que recibía $300 \mathrm{mg}$ versus el grupo que recibía placebo ( $\mathrm{p}<0,001$ para todas las comparaciones del cambio porcentual medio en el grupo de canakinumab versus el grupo placebo). Efectos similares fueron observados para el nivel de IL-6 (medido hasta los 12 meses). Por el contrario, el uso de canakinumab no redujo el nivel de LDL-C o HDL-C e incluso se observó $4 \%$ a $5 \%$ de aumento en el nivel medio de triglicéridos ${ }^{(2-4)}$.

En una mediana de seguimiento de 3,7 años la tasa de incidencia anual del evento primario fue de $4,11 \%$ en el grupo de $50 \mathrm{mg}$, versus $3,86 \%$ en el grupo de $150 \mathrm{mg}$, versus 3,90\% en el grupo de $300 \mathrm{mg}$, versus 4,50\% en el grupo placebo. El hazard ratio (HR) en relación con placebo fue 0,93 (IC 95\% 0,80-1,07; p=0,30) en el grupo de $50 \mathrm{mg} ; 0,85$ (IC $95 \%$ 0,74-0,98; $\mathrm{p}=0,021$ ) en el grupo de $150 \mathrm{mg}$ y 0,86 (IC 95\% 0,75-0,99; $\mathrm{p}=0,031$ ) en el grupo de $300 \mathrm{mg}$. La dosis de $150 \mathrm{mg}$ fue la única en alcanzar una diferencia significativa en el objetivo primario y secundario que incluye internación por angina inestable con revascularización urgente (HR vs placebo, 0,83; IC 95\% 0,73 a 0,95; $\mathrm{p}=0,005)$. No hubo diferencias significativas en mortalidad de cualquier $\operatorname{causa}^{(2-4)}$.

En cuanto a los efectos adversos, la neutropenia y las infecciones fatales fueron más frecuentes en los pacientes que recibieron canakinumab (tasa de incidencia anual de $0,31 \%$ vs $0,18 \%, p=0,02)$, especialmente en los pacientes más añosos y con diabetes. La trombocitopenia también fue más frecuente en los pacientes que recibieron canakinumab, pero sin aumento significativo en la incidencia de hemorragias. Se reportaron menos gota y osteoartritis en el grupo canakinumab, un efecto consistente con su inhibición de IL-1 $\beta$. Llamativamente, la mortalidad por cáncer fue menor en el grupo canakinumab versus placebo, principalmente ciertos cánceres de pulmón ${ }^{(2-4)}$.

Los autores concluyen que el tratamiento antiinflamatorio anti IL-1 $\beta$ con canakinumab en una dosis de $150 \mathrm{mg}$ cada tres meses reduce significativamente la tasa de eventos cardiovasculares comparado con placebo, independientemente de la reducción del nivel lipídico ${ }^{(2-4)}$.

Tras la presentación, el Dr. Eugene Braunwald (Hospital Brigham and Women's, Boston, MA) se mostró entusiasmado y manifestó a theheart.org| Medscape Cardiology: "Este fue un momento histórico... he venido a todas las reuniones desde que comenzó este encuentro del ESC en la década de 1960, no me he perdido ni una, y creo que este es probablemente el trabajo más importante que he escuchado en estos encuentros, porque muestra una nueva dirección de tra- 
tamiento para la afección cardíaca más importante, la aterosclerosis". Y añadió: "Todos hemos estado persiguiendo el LDL-Cy con los nuevos PCSK9 se reduce el LDL aún más y eso está bien. Pero lo que han hecho con este trabajo es demostrar que existe otro recurso"(5).

Uno de los principales problemas de este fármaco, más allá de consideraciones clínicas o fisiopatológicas, es su altísimo costo. Actualmente se comercializa a un precio de 200.000 dólares/año (administrado mensualmente). Este precio podría ser asumible para enfermedades raras, pero dada la alta prevalencia de la enfermedad cardiovascular sería insostenible la generalización de este tratamiento en la población y a priori parece que su relación costo-efectividad no es beneficiosa, como señala el Dr. Robert A. Harrington (Stanford University, Stanford, CA) en el editorial que acompaña al artículo ${ }^{(6)}$.

\section{Global Anticoagulant Registry in the Field - Atrial Fibrillation (Registro Global de Anticoagulantes en el Campo de la Fibrilación Auricular): GARFIELD-AF}

El registro GARFIELD-AF es una iniciativa de investigación académica independiente a cargo de un comité directivo internacional que cuenta con los auspicios del Thrombosis Research Institute (TRI) de Londres y con los fondos de una beca de investigación de Bayer Pharma AG (Berlín) ${ }^{(7)}$.

Se trata de un registro internacional, multicéntrico (al menos 1.000 centros en 35 países de América, Europa Occidental y Oriental, Asia, África y Australia), y observacional de pacientes con fibrilación auricular (FA) de diagnóstico reciente. El reclutamiento de pacientes, que comenzó en diciembre de 2009 y se extendió hasta agosto de 2015, logró recabar los datos de 57.262 pacientes en cinco cohortes prospectivas y secuenciales, con un período de seguimiento mínimo de dos años y máximo de ocho años, y actualmente casi dos años de seguimiento completo en las cinco cohortes $^{(8)}$.

Los pacientes incluidos debían haber sido diagnosticados de FA no valvular (FANV) en las últimas seis semanas y mostrar al menos un factor de riesgo adicional para accidente cerebrovascular (ACV), de tal forma que fueran potenciales candidatos para recibir tratamiento anticoagulante. Se dejó a juicio del investigador la identificación del o los factores de riesgo para $\mathrm{ACV}$, que no necesariamente se restringieron a los que se incluyen en los puntajes de riesgo establecidos. Los pacientes fueron incluidos, recibieran o no anticoagulación oral (ACO), de manera que las estrategias de tratamiento actuales o futuras y sus respectivas fallas pudieran ser reconocidas de forma adecuada en relación con los perfiles de riesgo individuales de cada paciente ${ }^{(7)}$.

La importancia de los datos recabados en este registro en torno a la carga real de la enfermedad, su tratamiento y el impacto de la terapia anticoagulante en las complicaciones tromboembólicas y hemorrágicas radica en que ponen de manifiesto el manejo en el mundo real de casi 60.000 pacien- tes y han sido presentados en múltiples congresos desde el 2015 a la fecha ${ }^{(7,8)}$.

En esta edición del ESC, los datos de este registro fueron presentados en el marco de un simposio denominado Atrial fibrillation - A bystander or cause of morbidity and mortality: insights from the GARFIELD-AF registry, auspiciado por Thrombosis Research Institute (TRI) de Londres $^{(8)}$.

El Dr. A. K. Kakkar (Gran Bretaña) presentó los datos globales acerca del uso de los recursos sanitarios en estos pacientes: GARFIELD-AF landmark: first-year outcomes of 52.000 prospective patients $^{(8,9)}$.

Una de las características más relevantes de este registro, por la manera en que fueron reclutados los pacientes en las distintas cohortes, es observar cómo han cambiado las estrategias de tratamiento a lo largo de los años: desde la cohorte 1 (2010-2011) con 57,4\% de pacientes en ACO versus $71,2 \%$ en la cohorte 5 (2015-2016) y cómo ha aumentado a su vez el uso de los nuevos anticoagulantes orales (NACO) desde $4,2 \%$ en la cohorte 1 a $43,1 \%$ en la cohorte 5 . Se ha visto también una disminución gradual en el uso de antiplaquetarios y lo que ha permanecido constante es el porcentaje de pacientes no tratados en alrededor de $10 \%$.

Con respecto a la incidencia anual en la tasa de eventos en este estudio prospectivo, destacó el riesgo de morir que tiene este grupo de pacientes, con una mortalidad por todas las causas de $4,38 \%$ (IC 95\% 4,20-4,57), ACV o embolismo sistémico de $1,36 \%$ (IC 95\% 1,26-1,47), sangrado mayor de 0,85\% (IC 95\% 0,77-0,94), síndromes coronarios agudos de $0,79 \%$ (IC 95\% 0,71-0,87), e insuficiencia cardíaca (IC) de 2,22\% (IC 95\% 2,09-2,35).

Respecto a las causas de muerte cardiovascular en este primer año de seguimiento, el 33,2\% fue por IC, muerte súbita $16 \%$, IAM 11,3\%, y el ACV isquémico fue la cuarta causa de muerte con $10,9 \%$. Y dentro de las muertes no cardiovascula- 
res, el $30,2 \%$ fue por malignidad y el $20,1 \%$ de causa infecciosa.

En cuanto al tipo de ACV, la gran mayoría corresponde a ACV isquémico $(69,4 \%)$, hemorragia intracerebral $(12,1 \%)$ y $18,5 \%$ indeterminado.

Al final de su presentación, el Dr. A. K. Kakkar puso énfasis en el adecuado manejo y tratamiento de todas las comorbilidades que acompañan a es- tos pacientes y no solo enfocarse en la FA per se, para de esta manera lograr los objetivos terapéuticos ${ }^{(9)}$.

Les recomendamos continuar viendo las siguientes presentaciones de este importante simposio en el link http://af.garfieldregistry.org/publications/2017-2/esc-final-congress-presentations.

\section{Estudio CASTLE- AF: Catheter ablation versus standard conventional treatment in patients with left ventricular dysfunction and atrial fibrillation}

Este estudio fue presentado por el Dr. Nassir Marrouche del Hospital de la Universidad de Utah, Salt Lake City, Estados Unidos. En su exposición el autor destacó que este es el único ensayo clínico aleatorizado que compara la ablación con catéter y la terapia farmacológica convencional en pacientes con insuficiencia cardíaca (IC) y fibrilación auricular (FA) donde se plantea como objetivo primario analizar la mortalidad y hospitalización por empeoramiento de $\mathrm{IC}^{(10)}$.

El CASTLE-AF tuvo un diseño prospectivo, multicéntrico, aleatorizado, controlado y fue realizado en 31 hospitales de Estados Unidos, Europa y Australia(11).

Se reclutaron 3.013 individuos, de los que se incluyeron solo 363 pacientes con FA y disfunción ventricular izquierda. Se dividieron en dos ramas: 184 pacientes recibieron tratamiento farmacológico convencional según las pautas actuales para FA en IC y a 179 pacientes se les realizó un protocolo de ablación con catéter aislando las venas pulmonares después de un intervalo de cinco semanas para optimizar el tratamiento de $\mathrm{IC}^{(10,11)}$.

El seguimiento fue de 37,8 meses (mediana). La media de edad fue de 64 años y la media de la fracción de eyección del ventrículo izquierdo (FEVI) fue de $35 \%$. Se incluyeron pacientes con FA paroxística o persistente sintomática, intolerancia o renuencia a tomar al menos un medicamento antiarrítmico, FEVI $\leq 35 \%$, clase funcional de la NYHA II, cardiodesfibrilador implantable (CDI) o terapia de resincronización cardíaca con desfibrilador con capacidad de monitoreo domiciliario ${ }^{(12)}$.

El punto final primario fue un compuesto de mortalidad por todas las causas u hospitalización por IC, el cual ocurrió en $28,5 \%$ de los pacientes del grupo con ablación versus 44,6\% del grupo con tratamiento convencional $(\mathrm{p}=0,007)$. La reducción del riesgo relativo del objetivo primario fue de $38 \%$ (HR $0,62$, IC $95 \% 0,43-0,87)^{(12)}$.

Los objetivos secundarios incluyeron mortalidad por todas las causas, hospitalización por empeo- ramiento de la IC, mortalidad cardiovascular y accidente cerebrovascular (ACV), así como calidad de vida, tolerancia al ejercicio, episodios de descarga del CDI, carga de FA e intervalos libres de $\mathrm{FA}^{(10)}$.

En el grupo de ablación de FA se observó una reducción de la mortalidad por todas las causas (HR 0,53 , IC $95 \% 0,32-0,86, p=0,011$ ), de las internaciones por empeoramiento de IC (HR 0,56, IC 95\% $0,37-0,83, p=0,0004)$, de la mortalidad cardiovascular (HR 0,49, IC 95\% 0,29-0,84, p=0,008) y de hospitalización por causa cardiovascular (HR 0,72, IC 95\% 0,52-0,99, $\mathrm{p}=0,041)^{(10)}$.

Los eventos adversos graves incluyeron derrame pericárdico ( 3 en el grupo de ablación versus 0 con tratamiento convencional); hemorragia aguda grave ( 3 en el grupo de ablación versus 0 con tratamiento convencional); ACV o accidente isquémico transitorio ( 7 en el grupo de ablación versus 12 con tratamiento convencional); estenosis de venas pulmonares ( 1 en el grupo de ablación versus 0 con tratamiento convencional); neumonía ( 3 en el grupo de ablación versus 1 con tratamiento convencional); infección inguinal ( 1 en el grupo de ablación versus 0 con tratamiento convencional), y empeoramiento de la IC ( 1 en el grupo de ablación versus 0 con tratamiento convencional)(10).

Los resultados finales muestran una reducción significativa en la mortalidad u hospitalización en aquellos pacientes con FA y disfunción ventricular izquierda a quienes se les realizó ablación con catéter en comparación con los que recibieron terapia farmacológica convencional ${ }^{(12)}$.

Para destacar lo innovador de su estudio, el autor principal refirió que "todos los estudios realizados sobre ablación en el pasado miran hacia la FA, pero esta es la primera vez que informamos puntos finales primarios duros relacionados con la ablación"(10).

En la presentación, el Dr. Marrouche mostró que el efecto en la mortalidad de la ablación versus el tratamiento convencional se hizo evidente aproximadamente a los tres años, mientras que la reduc- 
ción en la hospitalización por IC comenzó a ocurrir a los seis meses.

A 60 meses los datos de los dispositivos implantables indicaron que la carga de FA se redujo significativamente en los pacientes sometidos a ablación. El cambio de la FEVI basal también fue significativamente mayor en comparación al tratamiento convencional en el seguimiento a los 12 , 36 y 60 meses.
"A los cinco años tenemos un aumento de $8 \%$ en la FEVI versus ninguno en el grupo de tratamiento convencional, y es por eso que estamos muy entusiasmados", dijo Marrouche en una entrevista ${ }^{(10)}$.

Sin dudas, este estudio controversial fue uno de los más destacados del congreso europeo. Esperamos su publicación para poder realizar un análisis más profundo de sus datos.

\section{Cardiovascular OutcoMes for People using Anticoagulation StrategieS (COMPASS) trial: Primary Results}

El estudio fue presentado el pasado 27 de agosto por el Dr. John William Eikelboom de la Universidad McMaster, Hamilton, Canadá. Simultáneamente fue publicado online en The New England Journal of Medicine bajo el título: Rivaroxaban with or without Aspirin in Stable Cardiovascular Disease ${ }^{(13)}$.

El COMPASS fue diseñado para probar la hipótesis de que rivaroxabán en combinación con aspirina, o administrado solo, es más efectivo que aspirina en la prevención de eventos cardiovasculares recurrentes, con una seguridad aceptable, en pacientes con enfermedad vascular aterosclerótica esta$\mathrm{ble}^{(13)}$.

Es un estudio aleatorizado, doble ciego, realizado desde marzo de 2013 hasta mayo de 2016 y que incluyó 602 centros de 33 países. Se enrolaron 27.395 pacientes con enfermedad arterial aterosclerótica estable, en quienes se comparó rivaroxabán 2,5 mg dos veces al día más aspirina $100 \mathrm{mg}$ una vez al día $(\mathrm{N}=9.152)$, y rivaroxabán $5 \mathrm{mg}$ dos veces al día $(\mathrm{N}=9.117)$, o aspirina $100 \mathrm{mg}$ una vez al día $(\mathrm{N}=9.126)^{(13)}$.

La edad media fue de 68,2 años, $22,0 \%$ eran mujeres, $38 \%$ diabéticos y $75 \%$ hipertensos. Se incluyeron pacientes poscirugía de revascularización coronaria dentro de los primeros 4 a 14 días del posoperatorio. Se utilizaron agentes hipolipemiantes en $89,8 \%$ e inhibidores de la enzima convertidora de angiotensina o bloqueadores del receptor de angiotensina en 71,2\%. El 90,6\% tenía historia de enfermedad arterial coronaria y $27,3 \%$ tenía historia de enfermedad arterial periférica ${ }^{(13)}$.

Se excluyeron los pacientes con alto riesgo de sangrado, ACV reciente, ACV hemorrágico o lacunar previo, insuficiencia cardíaca grave, enfermedad renal estable avanzada (IFG $<15 \mathrm{ml} / \mathrm{min}$ ), terapia antiplaquetaria dual, ACO u otra terapia antitrombótica y condiciones no cardiovasculares de mal pronóstico, según la consideración del investigador ${ }^{(13)}$.

Una vez aleatorizados, los participantes fueron vistos al mes y a los seis meses; luego a intervalos semestrales. Debido a la superioridad del grupo de rivaroxabán más aspirina, el estudio se detuvo en forma prematura con una media de seguimiento de 23 meses $^{(13)}$.

El objetivo primario de eficacia fue la comparación de rivaroxabán con o sin aspirina versus aspirina sola mediante el punto final compuesto de muerte cardiovascular, ACV o infarto agudo de miocardio (IAM). Este punto final ocurrió en 379 pacientes $(4,1 \%)$ del grupo rivaroxabán más aspirina frente a $448(4,9 \%)$ en el grupo rivaroxabán solo, y 496 $(5,4 \%)$ de los asignados a aspirina sola. Para la comparación de rivaroxabán más aspirina con aspirina sola el HR para el punto final compuesto fue 0,76 (IC 95\%, 0,66-0,86; p <0,001). Para la comparación de rivaroxabán solo con aspirina sola el HR fue 0,90 $(\text { IC 95\%, 0,79-1,03; } \mathrm{p}=0,12)^{(13)}$.

El principal resultado de seguridad fue una modificación de los criterios de la Sociedad Internacional de Trombosis y Hemostasis para sangrado mayor e incluyó sangrado fatal, sangrado sintomático en un órgano crítico, sangrado en un sitio quirúrgico que requiere reintervención, y sangrado que requirió hospitalización. Los resultados mostraron que la modificación de estos criterios fue significativamente mayor en el grupo de rivaroxabán más aspirina comparado con aspirina sola(13).

Ocurrieron más eventos hemorrágicos mayores en pacientes del grupo de rivaroxabán más aspirina que en el grupo de aspirina sola, 288 pacientes $(3,1 \%)$ vs 170 pacientes $(1,9 \%)$ respectivamente (HR 1,70; IC 95\%, 1,40-2,05; p <0,001). La mayoría de los sangrados mayores fueron del tracto gastrointestinal, sin diferencia significativa entre los grupos en la tasa de hemorragia fatal, sangrado intracraneal o hemorragia sintomática en un órgano crítico.

También se registraron más eventos hemorrágicos mayores cuando se comparó el grupo de rivaroxabán solo con el grupo de aspirina (255 pacientes [2,8\%] vs 170 pacientes [1,9\%]; HR 1,51; IC 95\%, $1,25-1,84 ; \mathrm{p}<0,001)$. El exceso de sangrados mayo- 
res incluyó sangrado sintomático en un órgano crítico y sangrado que requirió hospitalización ${ }^{(13)}$.

Se reportaron $313(3,4 \%)$ muertes en el grupo de rivaroxabán más aspirina en comparación con 378 $(4,1 \%)$ en el grupo de aspirina (HR 0,82; IC 95\%, $0,71-0,96 ; \mathrm{p}=0,01 ;$ para un valor de $\mathrm{p}$ significativo de 0,0025$)^{(13)}$.

El análisis del beneficio clínico neto se realizó mediante un punto combinado de muerte, IAM, ACV, sangrado fatal o sangrado sintomático en un órgano crítico. Los resultados fueron a favor del grupo rivaroxabán más aspirina $(4,7 \%)$ frente al grupo de aspirina (5,9\%) (HR 0,80; IC95\%, 0,70-0,91; $\mathrm{p}<0,001)$. La comparación del grupo de rivaroxabán solo $(5,5 \%)$ versus aspirina no evidenció una diferencia significativa (HR, 0,94; IC 95\%, 0,84-1,07; $\mathrm{p}=0,36)^{(13)}$.

En conclusión, en pacientes con enfermedad vascular aterosclerótica estable el riesgo de padecer un evento cardiovascular mayor fue significativamente menor con la combinación de rivaroxabán más aspirina que con aspirina sola, a expensas de un aumento del riesgo de hemorragia mayor. El rivaroxabán administrado solo no disminuyó los eventos cardiovasculares en comparación con la aspirina sola y resultó en más eventos hemorrágicos mayores $^{(13)}$.

El Dr. J. W. Eikelboom dijo: "Los beneficios sustanciales observados con rivaroxabán y la aspirina respaldan el uso de dosis bajas de los dos tratamientos combinados. Ensayos recientes en otras áreas han demostrado beneficios sustanciales del uso de combinaciones de medicamentos a dosis bajas, y este concepto ahora está respaldado por los resultados de COMPASS"(14).

El profesor Stuart Connolly, coinvestigador principal y profesor de medicina de la Universidad McMaster, sugirió que el aumento del sangrado debería considerarse en el contexto de los hallazgos generales: "Muchas de estas hemorragias no fueron graves, y a pesar del aumento en el sangrado, los resultados muestran claramente un beneficio neto para los pacientes, como lo destaca la reducción del $18 \%$ en la mortalidad"(14).

Destacamos que en el COMPASS se realizó una doble aleatorización. Por un lado se efectuó el estudio que acabamos de comentar y simultáneamente se hizo otro ensayo que comparó pantoprazol versus placebo. El análisis de este trabajo excede a esta publicación dado que la investigación aún no está concluida.

\section{Bibliografía}

1. European Society of Cardiology. About ESC Congress. Disponible en: https://www.escardio.org/Congresses-\&-Events/ESC-Congress/Aboutthe-congress. [Consulta: 15 Oct 2017].

2. Ridker PM, Everett BM, Thuren T, MacFadyen JG, Chang WH, Ballantyne C, et al. Antiinflammatory Therapy with Canakinumab for Atherosclerotic Disease. N Engl J Med 2017; 377(12):1119-31.

3. Ridker PM, MacFadyen JG, Thuren T, Everett BM, Libby P, Glynn RJ, et al. Effect of interleukin-1 $\beta$ inhibition with canakinumab on incident lung cancer in patients with atherosclerosis: exploratory results from a randomized, double-blind, placebo-controlled trial. Lancet 2017; 390(10105): 183342 .

4. European Society of Cardiology. CANTOS: anti-infammatory drug cuts CV events, cancer. Presentation. ESC Congress 2017. Barcelona 24 - $30 \mathrm{Au}-$ gust, 2017.

5. Wendling $\mathbf{P}$. Coverage from the European Society of Cardiology (ESC) Congress 2017- Conference: CANTOS,anti-inflammatory drug cuts CV events, cancer. Medscape, August 27, 2017. Disponible en: https://www.medscape.com/viewarticle/884745. [Consulta: 15 Oct 2017 ].

6. Harrington RA. Targeting Inflammation in Coronary Artery Disease. N Engl J Med 2017; 377(12): 1197-8.

7. Thrombosis Research Institute. Global Anticoagulant Registry in the Field. Real-world insights from GARFIELD-AF Registry presented at ESC Congress 2017. London: TRI, 2017. Disponible en: http://af.garfieldregistry.org/wp-content/uploads/ 2017/08/ESC-Backgrounder-2017.pdf. [Consulta: 15 oct 2017 ]

8. European Society of Cardiology. ESC final congress presentations: symposium presentations. Disponible en:http://af.garfieldregistry.org/publications/2017-2/esc-final-congress-presentations. [Consulta: 5 nov 2017 ].

9. Kakkar A. Atrial fibrillation - A bystander or cause of morbidity and mortality: insights from the GARFIELD-AF registry. ESC Congress 2017. Barcelona 24 - 30 August, 2017. Disponible en: https://www.youtube.com/embed/u0tO84vjz4c?rel= 0\&showinfo $=0$.[Consulta: 5 nov 2017 ].

10. Jeffrey S. CASTLE-AF: ablation reduces mortality in LV dysfunction, AF. Medscape, August 29, 2017. Disponible en: https://www.medscape.com/viewarticle/884930. [Consulta: 5 Nov 2017].

11. Tomas L. Estudio CASTLE-AF: Ablación por catéter comparada con la terapia convencional en pacientes con fibrilación auricular y disfunción ventricular izquierda. Buenos Aires: SAC, 2017. Disponible en: 
http://www.sac.org.ar/esc-2017/estudio-castle-af-ablacion-por-cateter-comparada-con-la-terapia-convencional-en-pacientes-con-fibrilacion-auricular-y-disfuncion-ventricular-izquierda/.[Consulta: 5 Nov 2017].

12. American College of Cardiology. CASTLE-AF: catheter ablation vs. conventional therapy for patients with AFib and LV dysfunction. Washington, DC: ACC, 2017. Disponible en: http://www. acc.org/latest-in-cardiology/articles/2017/08/16/13/ 24/sun11am-castle-af-catheter-ablation-conventional-therapy-patients-afib-lv-dysfunction-esc-2017? _ga $=2.182341095 .1980941262 .1509477077-153645$ 0647.1509477077. [Consulta: 5 Nov 2017].
13. Eikelboom JW, Connolly SJ, Bosch J, Dagenais GR, Hart RG, Shestakovska O, et al; COMPASS Investigators. Rivaroxaban with or without Aspirin in Stable Cardiovascular Disease. N Engl J Med 2017; 377(14):1319-30.

14. European Society of Cardiology. Rivaroxaban plus aspirin improves outcomes in stable cardiovascular disease (COMPASS). Barcelona, August 27 2017. Disponible en:https://www.escardio.org/TheESC/Press-Office/Press-releases/rivaroxaban-plusaspirin-improves-outcomes-in-stable-cardiovascular-disease-compass?_ga=2.12496308.1868168912. 1510081611-1138021730.1510081611. [Consulta: 5 Nov 2017]. 